\title{
Three cases of amoebic liver abscess causing inferior vena cava obstruction, with a review of the literature
}

\author{
Anil K Sarda, Rakesh Mittal, Baljeet K Basra, Anurag Mishra, and Nikhil Talwar \\ Department of Surgery, Lok Nayak Hospital, Maulana Azad Medical College, New Delhi, India
}

\begin{abstract}
Amoebic liver abscess is a common disease, especially in endemic areas, but it is a rare cause of inferior vena cava (IVC) obstruction, with only a few cases appearing in the literature. We report three cases of amoebic liver abscess complicated with obstruction of the IVC and which responded to conservative treatment or radiological intervention. (Korean $\mathrm{J}$ Hepatol 2011;17:71-75)
\end{abstract}

Keywords: Liver abscess; Amoebiasis; Inferior vena cava; Obstruction

\section{INTRODUCTION}

Amoebiasis is the second leading cause of death from parasitic disease worldwide. ${ }^{1}$ Amoebic liver abscess (ALA) is the most common extra-intestinal manifestation of Entamoeba histolytica infestation, and it is associated with significant morbidity and mortality. ${ }^{2}$ The common complications of ALA include rupture into pleural cavity and peritoneal cavity; rarely, vascular complications are seen in ALA in the form of thrombosis or compression resulting in either hepatic venous outflow obstruction or inferior vena cava (IVC) obstruction. There have been only a few case reports of ALA with IVC obstruction. ${ }^{3-7}$ We present three cases of ALA where the patients presented with signs of IVC obstruction and radiological confirmation of the IVC obstruction by thrombosis and/or external compression.

\section{CASE REPORT}

\section{Case 1}

A24-year old man presented with pain in right upper abdomen with fever for five days. Physical examination revealed high grade fever and tachycardia. There was tenderness and guarding in right hypochondrium. There was bilateral pedal edema. Hematological investigations revealed a hemoglobin $(\mathrm{Hb})$ level of $11.2 \mathrm{~g} / \mathrm{dL}$ and white blood cell (WBC) count of $18,400 / \mathrm{mm}^{3}$ (normal range, $4,000-11,000 / \mathrm{mm}^{3}$ ). The biochemical investigations showed a blood urea nitrogen (BUN) of $34 \mathrm{mg} / \mathrm{dL}$ (normal range, $15-40 \mathrm{mg} / \mathrm{dL}$ ), serum creatinine of $1.1 \mathrm{mg} / \mathrm{dL}$ (normal range, $<1 \mathrm{mg} / \mathrm{dL}$ ). Liver functions tests showed a total bilirubin of $1.5 \mathrm{mg} / \mathrm{dL}$ (normal range, $<1 \mathrm{mg} / \mathrm{dL}$ ), serum alkaline phosphatase (ALP) of $178 \mathrm{IU} / \mathrm{L}$ (normal range, 40-280 IU/L), aspartate aminotransferase (AST) $32 \mathrm{IU} / \mathrm{L}$ (normal range, $<40$ IU/L), alanine aminotransferase (ALT) $48 \mathrm{IU} / \mathrm{L}$ (normal range, $<40 \mathrm{IU} / \mathrm{L}$ ). Chest X-ray was normal. Ultrasound at admission showed a large $(8.6 \times 7.3 \times 6.2 \mathrm{~cm})$, volume $202 \mathrm{~mL}$, heterogeneously hypoechoic lesion with internal echoes in the caudate lobe suggestive of liver abscess. On ultrasonography, the abscess was compressing the intra-hepatic IVC with abnormal color fill (slowed down) and flow pattern. Magnetic resonance imaging (MRI) with magnetic resonance cholangiopancreatography (MRCP) was performed which confirmed $6.9 \times 6 \mathrm{~cm}$ abscess in the caudate lobe causing eccentric compression over the intrahepatic part of IVC (Fig. 1, Fig. 2). There was a mass effect with

Received August 9, 2010; Revised November 23, 2010; Accepted December 9, 2010

Abbreviations: ALA, amoebic liver abscess; IVC, inferior vena cava; WBC, white blood cell; BUN, blood urea nitrogen; ALP, alkaline phosphatase; AST, aspartate aminotransferase; ALT, alanine aminotransferase; MRI, magnetic resonance imaging; MRCP, magnetic resonance cholangiopancreatography; ELISA, enzyme-linked immunosorbent assay

Corresponding author: A.K. Sarda

Department of Surgery, MAMC and Lok Nayak Hospital, New Delhi 110002, India

Tel. +0091-11-26011655, FAX. +0091-11-26672594, E-mail; aksarda@rediffmail.com 
compression of the main portal vein and the common hepatic duct just after the ductal confluence, however, the right and left hepatic ducts and intra-hepatic biliary radicals were not dilated. The patient tested positive for amoebic antibody by enzymelinked immunosorbent assay (ELISA) test. The patient was started on broad spectrum antibiotics and metronidazole for two weeks. After initiating the treatment, there was a gradual amelioration in the fever and abdominal pain. Repeat ultrasound by the end of first week revealed a gradual reduction in the size of the abscess to $50 \%$ of its original size and the compression on IVC was also relieved as confirmed by doppler scan. The patient was asymptomatic. Follow-up investigations showed WBC of $8,800 / \mathrm{mm}^{3}$, and liver

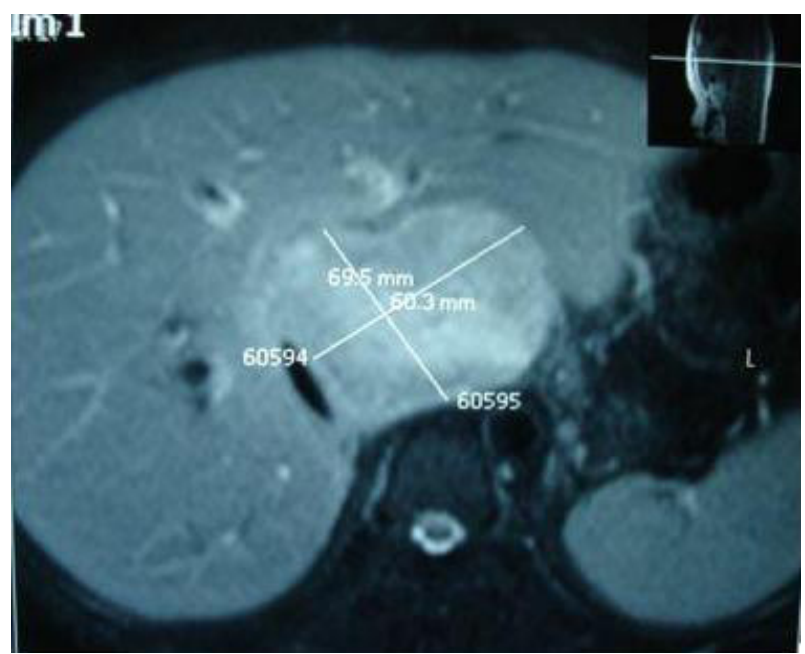

Figure 1. MRI of the abdomen showing a $6.9 \times 6.0 \mathrm{~cm}$ amoebic abscess in the caudate lobe of the liver causing extrinsic compression of the inferior vena cava.

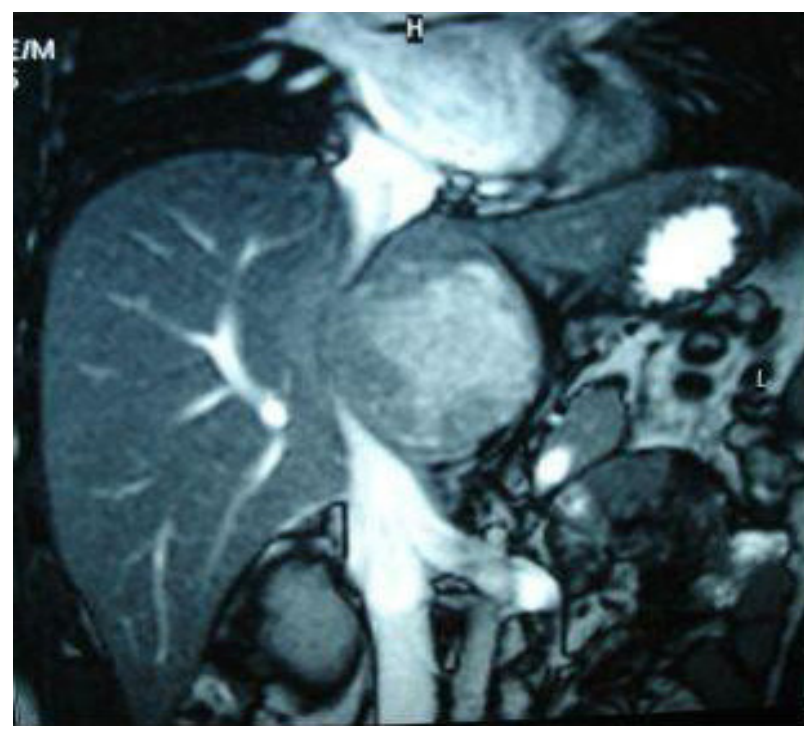

Figure 2. Coronal MRI showing an abscess in the caudate lobe of the liver causing extrinsic compression of the intrahepatic part of the inferior vena cava. functions tests showed a total bilirubin of $0.5 \mathrm{mg} / \mathrm{dL}$, serum ALP of $145 \mathrm{IU} / \mathrm{L}$, AST $40 \mathrm{IU} / \mathrm{L}$, ALT $45 \mathrm{IU} / \mathrm{L}$. Patient was followed up for three months and repeat ultrasound done at the end of three months showed no residual abscess and IVC was normal in caliber, color fill and flow pattern.

\section{Case 2}

A 21-year old male presented with upper abdominal pain for 15 days accompanied by a gradually increasing abdominal girth and swelling of the feet with low grade fever for one week. On examination, he was normo-tensive and showed pitting pedal edema. There was a tender hepatomegaly extending three fingers below the costal margin in the mid-clavicular line. Shifting dullness was positive. Hematological investigations revealed $\mathrm{Hb}$ level of $9 \mathrm{~g} / \mathrm{dL}$ and $\mathrm{WBC}$ of $7,500 / \mathrm{mm}^{3}$. The biochemical investigations showed a raised BUN of $65 \mathrm{mg} / \mathrm{dL}$ and serum creatinine of $1.5 \mathrm{mg} / \mathrm{dL}$. Urine analysis revealed slight proteinuria. Liver functions tests showed a total bilirubin of $0.6 \mathrm{mg} / \mathrm{dL}$, serum ALP of 247 IU/L, AST 40 IU/L and ALT 80 IU/L. Ultrasonography of the abdomen showed an abscess in the right lobe of liver on its postero-superior surface measuring $1.4 \times 1 \times 1 \mathrm{~cm}$ with internal echoes and moderate ascites. Color doppler scan was done which showed patent hepatic veins with normal hepatofugal flow. Hepatic IVC was seen displaced and compressed by the large abscess cavity but the flow was normal. Infra-hepatic IVC was dilated to $1.7 \mathrm{~cm}$. The Doppler findings were suggestive of IVC obstruction due to the liver abscess with proximal dilatation. A contrast enhanced CT scan confirmed the findings of thrombus in the IVC (Fig. 3). The patient tested positive for amoebic antibody by indirect haemagglutination test. The patient was started on intravenous metronidazole and

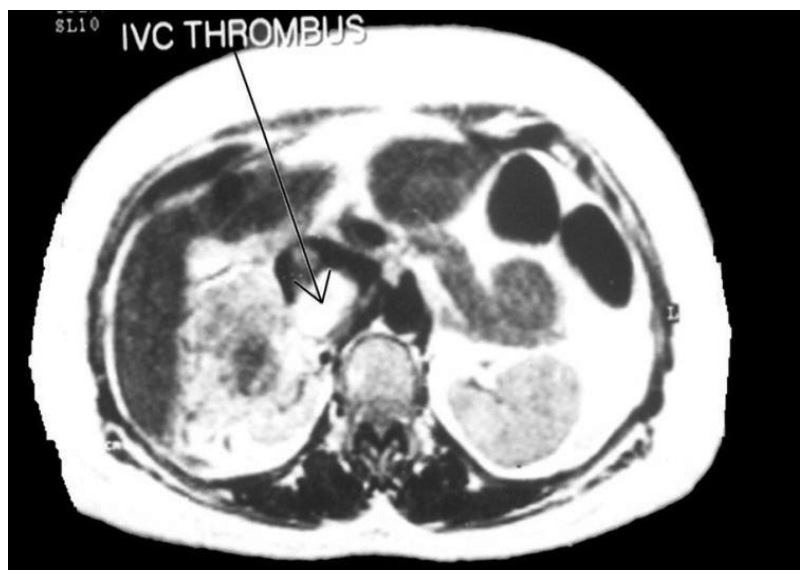

Figure 3. Contrast-enhanced CT of the abdomen showing an amoebic liver abscess with thrombus in the inferior vena cava. 
broad spectrum antibiotics. The liver abscess was aspirated twice under ultrasonic guidance within a period of two days yielding $500 \mathrm{~mL}$ and $360 \mathrm{~mL}$ of pus. The pus was thick, creamy and brownish in color and did not grow any organisms on culture. The ascites and pedal edema disappeared rapidly after the treatment. Doppler scan repeated four days later showed a decrease in the infra-hepatic IVC dilatation. Hepatic portion of the IVC was normal in caliber and fully patent in subsequent color doppler examination performed twelve days after the aspiration of pus. The biochemical renal parameters improved and two weeks after aspiration of the liver abscess, the BUN was 32 $\mathrm{mg} / \mathrm{dL}$ and serum creatinine was $1.1 \mathrm{mg} / \mathrm{dL}$. Patient was followed up for three months, and repeat ultrasonography done was essentially normal.

\section{Case 3}

A 61-year old man presented with high grade fever for eight days, vomiting for five days associated with right upper abdomen pain, yellowish discoloration of eyes for three days, and swelling of the feet for three days. He was icteric with a pulse rate of $96 / \mathrm{min}$ and blood pressure of 126/80 mmHg. There was bilateral pedal edema. On abdominal examination, tender hepatomegaly was present three finger breadth below the costal margin. Ultrasonography revealed multiple abscesses in the liver, the largest one $8 \times 7.2 \times 6.8 \mathrm{~cm}$ in the right lobe with mild pleural effusion. Hematological investigations revealed a $\mathrm{Hb}$ of $9.4 \mathrm{gm} / \mathrm{dL}$ and $\mathrm{WBC}$ $29,900 / \mathrm{mm}^{3}$ with predominantly polymorphic leukocytosis. The biochemical investigations showed a raised BUN of 59 $\mathrm{mg} / \mathrm{dL}$, serum creatinine of $1.2 \mathrm{mg} / \mathrm{dL}$. Liver function tests showed total bilirubin of $4.9 \mathrm{mg} / \mathrm{dL}$, direct bilirubin of 3.8

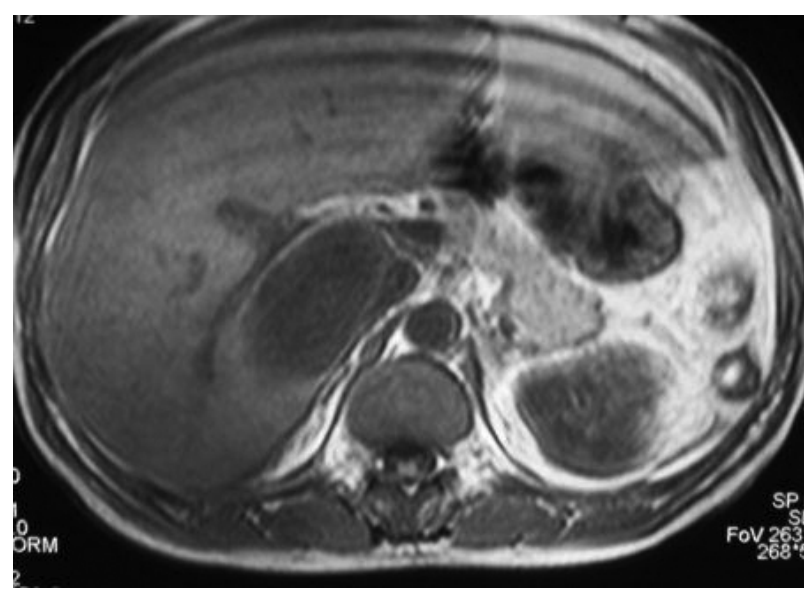

Figure 4. MRI of the abdomen showing multiple abscesses in the liver, the largest $(7.5 \times 7.2 \times 7.0 \mathrm{~cm})$ of which was in the right lobe. $\mathrm{mg} / \mathrm{dL}, \mathrm{ALP}$ of 1,055 IU/ L, AST of $99 \mathrm{IU} / \mathrm{L}$ and ALT of 108 IU/L. The patient tested positive for amoebic antibody by ELISA test. MRI with MRCP revealed four abscesses, two in the right lobe, one in the left lobe and one in the caudate lobe (Fig. 4). The largest one was $7.5 \times 7.2 \times 7 \mathrm{~cm}$ in size, in the right lobe. The abscess in the caudate lobe was compressing the porta hepatis and IVC (Fig. 5). The flow in IVC was found to be slowed down on color doppler. Catheter drainage was done from the largest abscess and the patient was started on intravenous metronidazole and broad spectrum antibiotics. Total $580 \mathrm{~mL}$ of pus was drained before the catheter was removed. After five days of admission the pedal edema reduced markedly and after ten days it subsided completely. Liver function tests returned to normal range with total bilirubin $1.1 \mathrm{mg} / \mathrm{dL}$, direct bilirubin $0.3 \mathrm{mg} / \mathrm{dL}$, ALP $22 \mathrm{IU} / \mathrm{L}$, AST $42 \mathrm{IU} / \mathrm{L}$ and ALT $20 \mathrm{IU} / \mathrm{L}$. The patient was discharged in good condition and followed up for three months and repeat ultrasound done at the end of three months showed no residual abscess and IVC was normal in caliber, color fill and flow pattern.

\section{DISCUSSION}

Amoebiasis occurs in $10 \%$ of the world's population and is most common in tropical and subtropical regions. ${ }^{1}$ Humans are the principal hosts and reservoir and get infected by ingestion of contaminated food and water containing amoebic

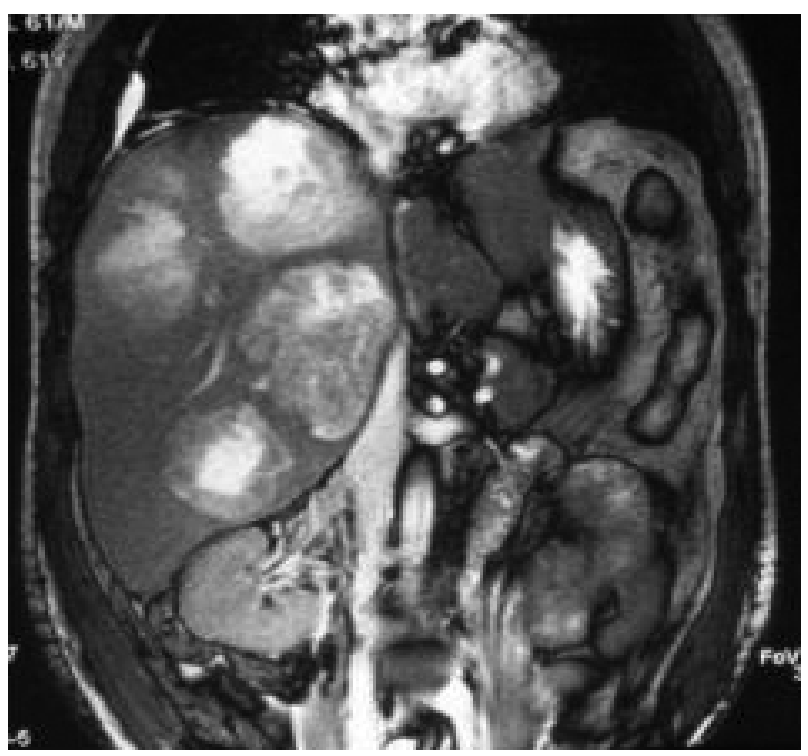

Figure 5. Coronal MRI showing multiple liver abscesses, with that in the caudate lobe causing extrinsic compression of the inferior vena cava. 
cysts. $^{2}$ ALA develops in less than $1 \%$ of patients infested with $E$. histolytica, but this still represents a large number of patients. ${ }^{2}$ The disease should be suspected in anyone with an appropriate exposure history (residency or travel in an endemic area) and fever, right upper quadrant pain, and substantial hepatic tenderness.

The diagnosis of ALA relies on identification of space occupying lesion of the liver and positive amoebic serology. ${ }^{1}$ Amoebic serology is highly sensitive (>94\%) and highly specific (>95\%) for the diagnosis of amoebic liver abscess. ${ }^{8}$ Antibodies develop in 90 to $100 \%$ of patients with ALA and become detectable at seven to ten days after the onset of symptoms. Indirect hemagglutination and enzyme-linked immunosorbent assay are widely used because of their high sensitivity. ${ }^{2}$ Computed tomography is an ideal tool to detect liver abscesses, particularly smaller lesions and its associated complications. Its sensitivity for detecting liver abscess is as high as $97 \%{ }^{9}$

The rate of various complications described in an ALA was reported to be $10.3 \%{ }^{10}$ Complications associated with liver abscess include rupture into pleural, pericardial \& peritoneal cavity, vascular thrombosis, and rupture into the bile ducts. ${ }^{11}$ Early diagnosis and prompt treatment are essential to reduce the morbidity and mortality associated with a liver abscess. The mortality rate being low when the abscess is limited to liver but it increases as the abscess gets complicated. Therefore, the early detection of complications associated with hepatic abscess is important. Vascular complications of hepatic abscess, such as thrombosis of the portal vein, occlusion of hepatic vein, or inferior vena cava obstruction, are rare and they have been previously described mostly in autopsy studies. ${ }^{12}$ This was a large study by Krishnan et al ${ }^{12}$ where retrospective analysis of 95 autopsies on ALA was done and IVC thrombosis was seen in $8 \%$ of the cases in combination with hepatic vein in all but one. One of the cases showed rupture of the abscess into IVC. Apart from this study, there have been very few case reports in which IVC obstruction has been described in patients with ALA. $^{3-7}$ Huddle $^{3}$ reported a case of 26 year old man with ALA where it was complicated with extrinsic compression of the IVC by an abscess in the caudate lobe of the liver at the level where it passes through the liver. There was no evidence of IVC thrombosis. Hodkinson et $\mathrm{al}^{4}$ reported another case of 50 year old man with ALA where the patient developed IVC obstruction due to a thrombus which extended up to the right atrium. Another case was reported by Sharma and $\mathrm{Sarin}^{5}$ where a 30 year old man presented with ALA with signs of hepatic venous outflow obstruction, later found to be due to compression of the IVC by a large and tense abscess in the caudate lobe of the liver. Sodhi et al $^{6}$ reported a case where amoebic liver abscess in a 57 year old man was complicated by hepatic vein and IVC thrombosis. Schmid et $\mathrm{al}^{7}$ reported another case of a 19 year old man with ALA where the patient had signs if IVC obstruction due to external compression of the IVC and not due to thrombosis. In another study by Yang et al, ${ }^{11}$ a retrospective analysis of 81 patients with pyogenic liver abscess, three patients had vascular complications. Of these three patients, two had portal vein thrombosis only and one had extrinsic compression of the IVC associated with portal vein thrombosis. There were only three cases of our report described where the ALA has led to extrinsic compression of the IVC in absence of any thrombosis so far. This complication can be managed effectively by catheter drainage or percutaneous needle aspiration combined with antibiotics. In all the three cases of ALA with IVC compression mentioned above, percutaneous needle aspiration of the abscess was required to relieve the compression and all the patients were discharged in good condition. In our cases, two patients required percutaneous drainage and in one patient, IVC compression was relieved with antibiotics alone. The management of ALA with IVC thrombosis again mainly includes antibiotics and drainage of the abscess, but in few cases, anticoagulation therapy has also been added to achieve complete resolution.

We report three cases of ALA causing IVC obstruction which completely resolved by the percutaneous drainage and appropriate antibiotic therapy.

\section{REFERENCES}

1. Stanley SL Jr. Amoebiasis. Lancet 2003;361:1025-1034.

2. Wells CD, Arguedas M. Amebic liver abscess. South Med J 2004; 97:673-682.

3. Huddle KR. Amoebic liver abscess, inferior vena-caval compression and the nephrotic syndrome. A case report. S Afr Med J 1982;61: 758-760.

4. Hodkinson J, Couper-Smith J, Kew MC. Inferior vena caval and right atrial thrombosis complicating an amebic hepatic abscess. Am J Gastroenterol 1988;83:786-788.

5. Sharma MP, Sarin SK. Inferior vena caval obstruction due to amoebic liver abscess. J Assoc Physicians India 1982;30:243-244.

6. Sodhi KS, Ojili V, Sakhuja V, Khandelwal N. Hepatic and inferior vena caval thrombosis: vascular complication of amebic liver abscess. J Emerg Med 2008;34:155-157. 
7. Schmid BD, Lalyre Y, Sigel B, Kiani R, Layden TJ. Inferior vena cava obstruction complicating amebic liver abscess. Dig Dis Sci 1982; 27:565-569.

8. Katzenstein D, Rickerson V, Braude A. New concepts of amebic liver abscess derived from hepatic imaging, serodiagnosis, and hepatic enzymes in 67 consecutive cases in San Diego. Medicine (Baltimore) 1982;61:237-246.

9. Halvorsen RA, Korobkin M, Foster WL, Silverman PM, Thompson WM. The variable CT appearance of hepatic abscesses. AJR Am J
Roentgenol 1984;142:941-946.

10. Adams EB, MacLeod IN. Invasive amebiasis. II. Amebic liver abscess and its complications. Medicine (Baltimore) 1977;56:325-334.

11. Yang DM, Kim HN, Kang JH, Seo TS, Park CH, Kim HS. Complications of pyogenic hepatic abscess: computed tomography and clinical features. J Comput Assist Tomogr 2004;28:311-317.

12. Krishnan K, Badarinath S, Bhusnurmath SR. Vascular complications of hepatic amoebiasis-a retrospective study. Indian J Pathol Microbiol 1986;29:293-296. 